Tendências em Matemática Aplicada e Computacional, 3, No. 1 (2002), 41-50.

(C) Uma Publicação da Sociedade Brasileira de Matemática Aplicada e Computacional.

\title{
Métodos de Pontos Interiores Aplicados a Problemas de Multifluxo com Restrições Adicionais
}

A.T. de AZEVEDO ${ }^{1}$, S. SOARES ${ }^{2}$, Faculdade de Engenharia Elétrica, Universidade Estadual de Campinas, 13083-970 Campinas, SP, Brazil.

A.R.L. de OLIVEIRA ${ }^{3}$, Instituto de Ciências Matemáticas e de Computação, 13560-970, São Carlos, SP, Brazil.

M.F.H. CARVALHO ${ }^{4}$, Laboratório de Tecnologia de Gestão Empresarial, Centro de Pesquisas Renato Archer, 13089-500, Campinas, SP, Brazil.

Resumo. O objetivo deste trabalho é formular e resolver o problema de planejamento e controle da manufatura pelo modelo de grafos, desenvolvendo métodos de pontos interiores que exploram em diferentes graus a esparsidade do problema. O desempenho computacional dos métodos é comparado para dados de problemas testes reais.

\section{Introdução}

A limitação de recursos materiais faz com que seja necessária a tomada de decisões sempre com o objetivo de aproveitar o máximo dos recursos disponíveis segundo um determinado critério.

Para os sistemas de manufatura, este processo envolve grande quantidade de variáveis. A utilização de modelos matemáticos para o auxílio nas tomadas de decisão pode garantir que seja obtida uma solução mais coerente com um determinado critério, além de tornar claras as considerações feitas para se tomar tal decisão.

A idéia central deste trabalho é utilizar um mesmo modelo matemático para os problemas de planejamento e controle da produção na manufatura de forma a se obter uma minimização dos custos de produção.

\section{Planejamento e Controle da Produção na Ma- nufatura}

Primeiramente, identificamos em um sistema real os pontos a serem representados pelo modelo e classificados como sugerido na Tabela 1.

\footnotetext{
1 anibal@densis.fee.unicamp.br

2dino@densis.fee.unicamp.br

3 aurelio@icmc.sc.usp.br

4Marcius.Carvalho@cenpra.gov.br
} 


\begin{tabular}{|c|l|c|c|}
\hline Sistema Produtivo & Entradas & Processo & Saídas \\
\hline Manufatura & Máquinas, & Fabricação e Montagem & Produtos \\
& Matéria-Prima, & & \\
& Mão-de-obra & & \\
\hline
\end{tabular}

Tabela 1: Características de processos produtivos.

Após a identificação dos elementos constituintes do sistema produtivo, estes devem ser vinculados a um modelo matemático que apresenta as seguintes características: o horizonte de planejamento é discretizado em períodos (hora, turno, dia); cada estágio de produção pode conter um ou mais grupos de máquinas; as capacidades de cada grupo e de armazenagem são definidas à priori dentro do horizonte de planejamento; os limites de oferta de matéria-prima para atendimento à demanda são conhecidos dentro do horizonte de planejamento.

O resultado de um método de resolução para o problema fornece um conjunto de decisões que caracterizam uma política de produção, incluindo: o quanto cada máquina deve produzir e em que período; o quanto armazenar por estágio e em que período; a escala de utilização de matéria-prima por período; a escala de atendimento à demanda por período; a parcela da demanda atendida com atraso; a parcela da demanda não atendida no horizonte de planejamento; o custo de produção dentro do horizonte de planejamento.

No caso da manufatura, podemos dizer que pelo sistema fluem itens, sendo que estes itens são matérias-primas sendo processadas por máquinas, ou sendo armazenadas, até se tornarem um produto.

Portanto, utilizamos a técnica de grafos para considerar a característica de itens fluindo pelo sistema com uma representação bastante intuitiva.

\section{O Modelo Matemático de Grafos}

Para visualizarmos como utilizar o modelo matemático de grafos, consideraremos um pequeno exemplo de Planejamento e Controle da Produção (PCP) na manufatura.

Imaginemos uma linha de produção com três células de produção com tarefas e número de máquinas distintos que devem fabricar um produto $\mathrm{P} 1$.

$\mathrm{O}$ produto $\mathrm{P} 1$ pode ser processado por qualquer máquina de cada célula para seguir para o próximo estágio da manufatura como descrito na Figura 1.

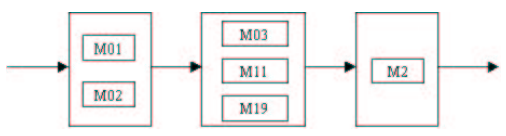

Figura 1: Representação da linha de produção de P1.

A Figura 1 representa uma linha de produção para um período de tempo. Para 
representar $n$ intervalos de tempo, basta replicar $n$ vezes a Figura 1 . Um exemplo disto é a Figura 2, onde replicamos a linha da Figura 1 para três períodos.

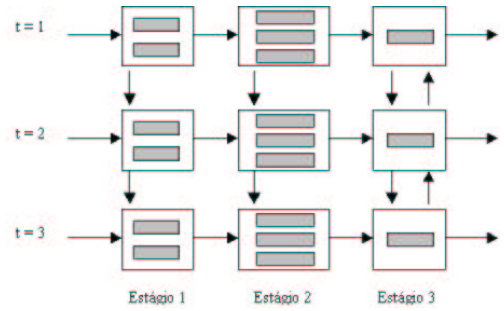

Figura 2: Sistema de produção com 3 estágios e 3 períodos.

Na Figura 2, a estocagem é representada pela transferência de produtos de um tempo $t$ para um tempo $t+1$, enquanto a entrega com atraso é modelada pela transferência de produtos do tempo $t$ para o tempo $t-1$ no último estágio.

O passo seguinte da modelagem matemática é transformar a Figura 2 no grafo da Figura 3. Os elementos do grafo da Figura 3 são descritos a seguir:

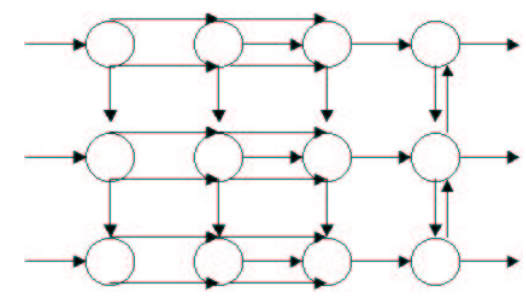

Figura 3: Representação por grafos do sistema de produção com 3 estágios e 3 períodos.

1. Arcos (setas): Simbolizam ação sobre um produto e são de duas naturezas:

- Horizontais: Indica o fluxo dos produtos na linha de montagem relativo ao fornecimento de matéria-prima ou em máquinas de uma célula, ou ainda, o atendimento de demanda de um produto para um mesmo período de tempo.

- Verticais: Indica o fluxo de produtos no tempo, seja com a estocagem de produtos, seja com o atendimento com atraso da demanda.

2. Nó (círculo): Ponto de tomada de decisão sobre um produto estocado (seta vertical) ou processado em uma máquina (seta horizontal) deve ser processado por uma das máquinas de uma dada célula (seta horizontal), ou se será estocado (seta vertical).

É importante observar que as características da rede em cada período podem variar de acordo com a capacidade das máquinas, disponibilidade de matéria-prima, capacidade de estocagem e atendimento de demanda com ou sem atraso. 
O último passo da modelagem é transformar a estrutura proposta na Figura 3 em uma representação manipulável, ou seja, uma matriz de incidência nó-arco $A$.

Um resumo das etapas realizadas pode ser visto na Figura 4.

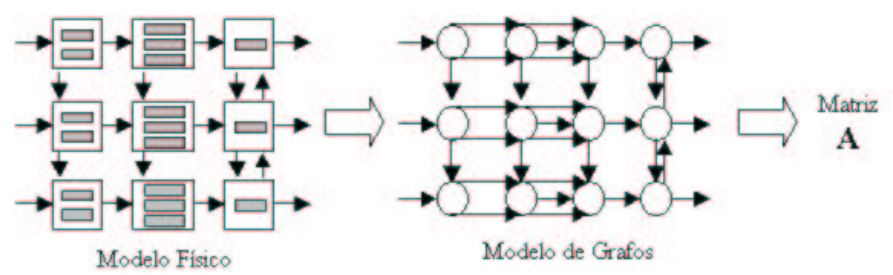

Figura 4: Etapas da modelagem do PCP na manufatura.

O modelo proposto até agora e que consta nas Figuras 2 e 3 supõe a existência de apenas um produto P1. Porém, é muito comum que em uma manufatura ocorra o processamento de mais de um produto, podendo existir dois casos:

Caso 1: Os produtos a serem manufaturados utilizam máquinas diferentes, portanto suas linhas de produção são independentes (não tem acoplamento). As etapas da modelagem ficam como na Figura 5.

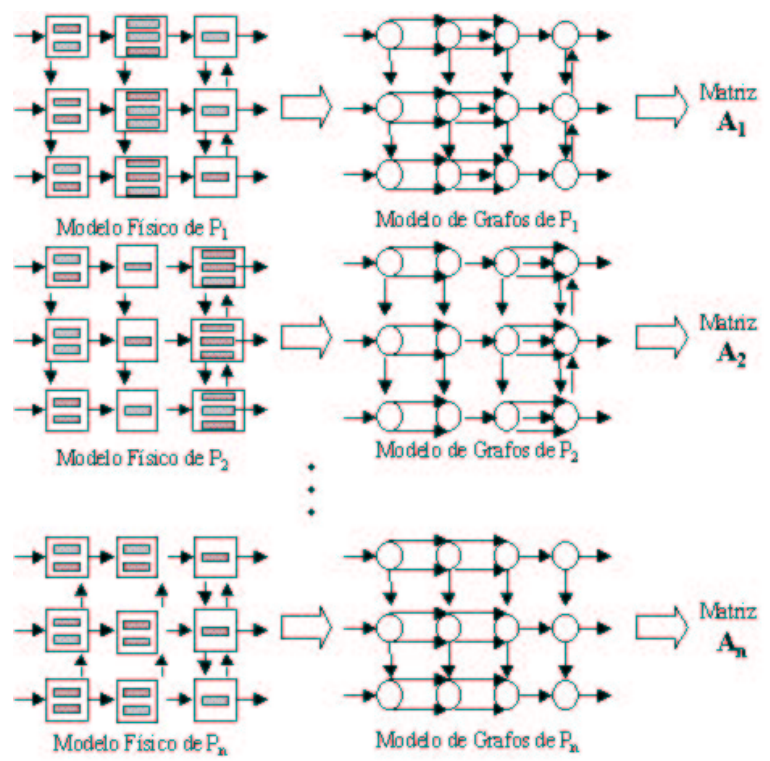

Figura 5: Etapas da modelagem do PCP na manufatura multiproduto sem acoplamento.

Como resultado de tal modelagem, temos o sistema (3.1). 


$$
\left[\begin{array}{cccc}
A_{1} & 0 & \cdots & 0 \\
0 & A_{2} & \cdots & 0 \\
\vdots & \vdots & \ddots & \vdots \\
0 & 0 & \cdots & A_{n}
\end{array}\right]\left[\begin{array}{c}
x_{1} \\
x_{2} \\
\vdots \\
x_{n}
\end{array}\right]=\left[\begin{array}{c}
b_{1} \\
b_{2} \\
\vdots \\
b n
\end{array}\right]
$$

Onde $b$ é um vetor cujos componentes $b_{i}$ representam a disponibilidade de matéria-prima e a demanda para cada produto $i$.

Caso 2: Os produtos a serem manufaturados utilizam máquinas em comum, tal que suas linhas de produção devem compartilhar recursos disponíveis (acoplamento). As etapas da modelagem ficam como na Figura 5, mas, agora, temos que representar em forma matricial a possibilidade de processamento de mais de um tipo de produto por uma ou mais máquinas. Assim, além do sistema (3.1), temos a restrição (3.2).

$$
S x \leq d \Rightarrow\left[\begin{array}{llll}
S_{1} & S_{2} & \cdots & S_{n}
\end{array}\right]\left[\begin{array}{c}
x_{1} \\
x_{2} \\
\vdots \\
x_{n}
\end{array}\right] \leq\left[\begin{array}{c}
d_{1} \\
d_{2} \\
\vdots \\
d_{m}
\end{array}\right] .
$$

Onde a matriz $S$ representa as restrições de acoplamento tal que cada máquina $j$ não ultrapasse sua capacidade $d_{j}$ ao processar $i$ produtos.

Por último, em ambos os casos as capacidades mínimas e máximas de cada máquina devem ser respeitadas, o que é representado por (3.3).

$$
\left[\begin{array}{c}
l_{1} \\
l_{2} \\
\vdots \\
l_{n}
\end{array}\right] \leq\left[\begin{array}{c}
x_{1} \\
x_{2} \\
\vdots \\
x_{n}
\end{array}\right] \leq\left[\begin{array}{c}
u_{1} \\
u_{2} \\
\vdots \\
u_{n}
\end{array}\right]
$$

Uma formulação matemática geral do nosso problema é:

$$
\begin{gathered}
\operatorname{Min} c^{t} x \\
\text { S. } a: A x=b \\
\text { Sx } \leq d \\
l \leq x \leq u,
\end{gathered}
$$

onde a função objetivo é relacionada aos custos de transporte ou processamento dos produtos, $l$ e $u$ representam os limites inferiores e superiores, respectivamente, dos valores de $x$.

Vale lembrar que, a matriz $A_{i}$ pode simbolizar uma rede associada a um produto para um dado período ou uma rede para um produto em vários períodos. Uma última observação é que os modelos propostos por $(3.1,3.2,3.3)$ e (3.4) permitem que tenhamos uma rede multiestágio, multiperíodo e multiproduto com acoplamento entre os produtos. Maiores detalhes sobre estes problemas em [2, 4]. 


\section{Resolução pelos Métodos de Pontos Interiores}

O problema multiproduto com acoplamento entre os produtos é comumente chamado de problema de multifluxo com restrições adicionais [2]. Estes problemas são freqüentemente modelados como problemas de programação linear.

Muitas vezes, problemas reais são de dimensões tão elevadas que a aplicação direta do método simplex se torna proibitiva [2]. Na verdade, as restrições de acoplamento tornam este problema de difícil resolução, exigindo a utilização de técnicas que procurem explorar a estrutura bloco angular da matriz $A$ [1].

Neste sentido, podemos utilizar os seguintes métodos: Método de Decomposição de Dantzig-Wolfe, Penalização Linear Quadrática, Método de Decomposição Simplicial, Método de Pontos Interiores. Uma comparação entre os 3 primeiros métodos pode ser vista em [5].

O propósito deste trabalho é o desenvolvimento de métodos de pontos de interiores (MPIs) que explorem a estrutura esparsa do problema de PCP na manufatura.

Portanto, consideramos 3 estruturas esparsas de $A$, e a partir de cada uma delas desenvolvemos os métodos de pontos de interiores específicos. As 3 estruturas de esparsas fornecem:

1. Sem considerar nenhuma estrutura particular.

2. Decompondo $A$ em matriz de atendimento de demanda $A$ dos $i$ produtos e em matriz de acoplamento $S$ : Formulação dada por (3.4).

3. Decompondo $A$ em $A_{i}$ matrizes e $S$ em $S_{i}$ matrizes para os $i$ produtos: Formulação dada por $(3.1,3.2,3.3)$.

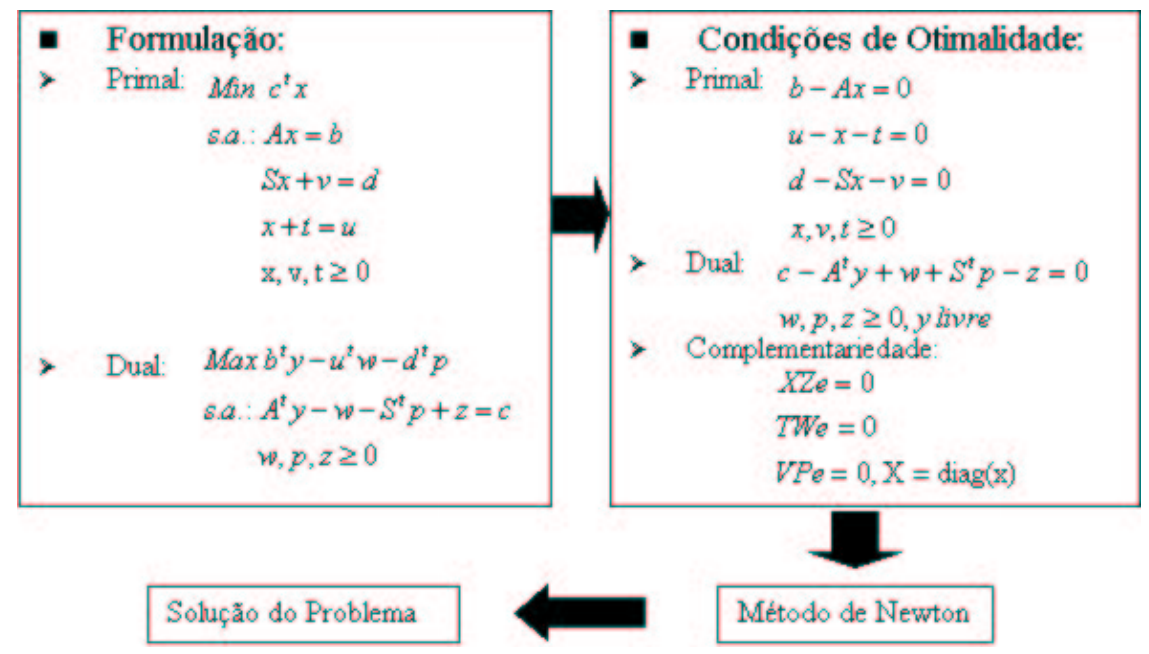

Figura 6: Construindo um MPI com estrutura esparsa $A$ e $S$. 
A essência de um MPI primal-dual consiste em aplicar o método de Newton às condições de otimalidade de um problema de programação linear [6]. As etapas necessárias para se construir um MPI associado ao problema dado por (3.4) estão na Figura 6. Os passos de um MPI, que considera estrutura esparsa $A$ e $S$, são detalhados a seguir.

Dados $\left(x^{0}, t^{0}, v^{0}, w^{0}, p^{0}, z^{0}\right)>0$ e $y^{0}$ livre e $\tau \in(0,1)$

Para $\mathrm{k}=0,1, \ldots$

$$
\begin{aligned}
& \mu^{k}=\delta \gamma / n_{p} \text {, onde: } n_{p}=\text { dimensão do vetor }(\widetilde{x}, t, v) \text { (GAP médio) } \\
& \Gamma_{p}^{k}=\widetilde{b}-A \widetilde{x}^{k}=b-A l-A \widetilde{x}^{k} \\
& \Gamma_{\widetilde{u}}^{k}=\widetilde{u}-\widetilde{x}^{k}-t^{k}=u-l-x^{k}-t^{k} \\
& \Gamma_{\widetilde{d}}^{u}=\widetilde{d}-S \widetilde{x}^{k}-v^{k}=d-S l-S x^{k}-v^{k} \\
& \Gamma_{d}^{k}=c-A^{t} y^{k}+w^{k}+S^{t} p^{k}-z^{k} \\
& \Gamma_{c}^{k} \quad=\mu e-X^{k} Z^{k} e \\
& \Gamma_{b}^{k}=\mu e-V^{k} P^{k} e \\
& \Gamma_{e}^{k} \quad=\mu e-T^{k} W^{k} e \\
& D^{k}=\left(\left(T^{k}\right)^{-1} W^{k}+S^{t}\left(V^{k}\right)^{-1} P^{k} S+\left(X^{k}\right)^{-1} Z^{k}\right) \\
& d y^{k}=\left(A\left(D^{k}\right)^{-1} A^{t}\right)^{-1}\left(\Gamma_{p}^{k}+A\left(D^{k}\right)^{-1}\left(\Gamma_{d}^{k}+\left(T^{k}\right)^{-1} \Gamma_{e}^{k}+S^{t}\left(V^{k}\right)^{-1} \Gamma_{b}^{k}-X^{-1} \Gamma_{c}^{k}\right.\right. \\
& \left.\left.-\left(T^{k}\right)^{-1} W^{k} \Gamma_{\widetilde{u}}^{k}-S^{t}\left(V^{k}\right)^{-1} P^{k} \Gamma_{\widetilde{d}}^{k}\right)\right) \\
& d x^{k}=\left(D^{k}\right)^{-1}\left(A^{t} d y^{k}-\Gamma_{d}^{k}-\left(T^{k}\right)^{-1} \Gamma_{e}^{k}-S^{t}\left(V^{k}\right)^{-1} \Gamma_{b}^{k}+X^{-1} \Gamma_{c}^{k}+\left(T^{k}\right)^{-1} W^{k} \Gamma_{\widetilde{u}}^{k}\right. \\
& \left.\left.+S^{t}\left(V^{k}\right)^{-1} P^{k} \Gamma_{\widetilde{d}}^{k}\right)\right) \\
& d t^{k}=\Gamma_{\sim}^{k}-d x^{k} \\
& d v^{k}=\Gamma_{\widetilde{d}}^{u}-S d x^{k} \\
& d z^{k}=(\stackrel{d}{X})^{-1}\left(\Gamma_{c}^{k}-Z^{k} d x^{k}\right) \\
& d p^{k}=\left(V^{k}\right)^{-1}\left(\Gamma_{b}^{k}-P^{k} d v^{k}\right) \\
& d w^{k}=\left(T^{k}\right)^{-1}\left(\Gamma_{e}^{k}-W^{k} d t^{k}\right) \\
& e_{p}^{k}=\operatorname{Min}\left\{\operatorname{Min}_{\partial x_{i}^{k}<0}\left(\frac{-x_{i}^{k}}{\partial x_{i}^{k}}\right), \operatorname{Min}_{\partial t_{i}^{k}<0}\left(\frac{-t_{i}^{k}}{\partial t_{i}^{k}}\right), \operatorname{Min}_{\partial v_{i}^{k}<0}\left(\frac{-v_{i}^{k}}{\partial v_{i}^{k}}\right)\right\} \\
& e_{d}^{k}=\operatorname{Min}\left\{\operatorname{Min}_{\partial w_{i}^{k}<0}\left(\frac{-w_{i}^{k}}{\partial w_{i}^{k}}\right), \operatorname{Min}_{\partial p_{i}^{k}<0}\left(\frac{-p_{i}^{k}}{\partial p_{i}^{k}}\right), \operatorname{Min}_{\partial z_{i}^{k}<0}\left(\frac{-z_{i}^{k}}{\partial z_{i}^{k}}\right)\right\} \\
& \alpha_{p}^{k} \quad=\operatorname{Min}\left(1, \tau e_{p}^{k}\right) \\
& \alpha_{d}^{k}=\operatorname{Min}\left(1, \tau e_{d}^{k}\right) \\
& x^{k+1}=x^{k}+\alpha_{p}^{k} d x^{k} \\
& t^{k+1}=t^{k}+\alpha_{p}^{k} d t^{k} \\
& v^{k+1}=v^{k}+\alpha_{p}^{k} d v^{k} \\
& y^{k+1}=y^{k}+\alpha_{d}^{k} d y^{k} \\
& z^{k+1}=z^{k}+\alpha_{d}^{k} d z^{k} \\
& p^{k+1}=p^{k}+\alpha_{d}^{k} d p^{k} \\
& w^{k+1}=w^{k}+\alpha_{d}^{k} d w^{k} \\
& k \leftarrow k+1
\end{aligned}
$$

Até Convergir.

Aqui $e$ é um vetor unitário de dimensões apropriadas, $X, Z, T, W, V$ e $P$ são matrizes diagonais, tais que $X=\operatorname{diag}(x)$, por exemplo. 
Cabe destacar que na inversão de $A\left(D^{k}\right)^{-1} A^{t}$, para o cálculo da direção $d y^{k}$, está a maior parte do esforço computacional do método. Mas, dado que a matriz $A$ é $m \times n, m<n$, tendo $m$ linhas linearmente independentes, então, $A\left(D^{k}\right)^{-1} A^{t}$ é simétrica, definida positiva e, na prática, usamos decomposição de Cholesky para resolver o sistema linear. Os valores dos parâmetros $\mu, \tau$ e $\sigma$ utilizados no método serão vistos na seção de resultados numéricos.

Além disso, construímos para cada estrutura esparsa, o método primal-dual e o método preditor-corretor. O preditor-corretor pode ser obtido com uma pequena modificação em relação ao primal-dual, com pequenos custos computacionais adicionais, mas com ganho no número total de iterações $[1,6]$.

\section{Resultados Numéricos}

Para testes dos algoritmos foram utilizados arquivos de dados de PCP na manufatura relativos ao Projeto Industrial Platform to Research, Teaching and Formation in Automation (PIPEFA) da Faculdade de Engenharia Mecânica da UNICAMP [3].

Como ilustração, o grafo associado ao problema Exemp2 está na Figura 7 e as demais redes são construídas de forma análoga.

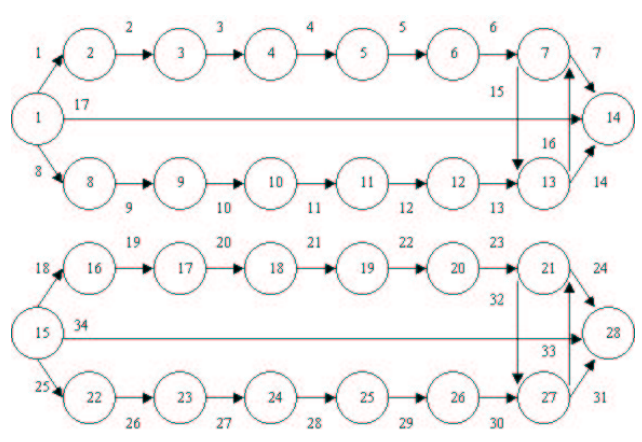

Figura 7: Grafo associado ao PCP na manufatura do problema teste Exemp2.

Ao aplicarmos os MPIs primal-dual e preditor-corretor para as 3 estruturas esparsas de $A$, vistas anteriormente, testamos os algoritmos para os seguintes parâmetros:

1. Primal-Dual:

- $\mu^{k}=\delta \gamma / n_{p}$ ou $\left\{\begin{array}{c}\mu^{k}=\delta\left(\gamma^{k}\right)^{2} / n_{p}, \text { se } \gamma^{k}<1 \\ \mu^{k}=\delta \gamma^{k} / n_{p}, \text { caso contrário }\end{array}\right\}$

- $\tau=0.995$ ou 0.99995

- $\sigma=0$ ou $1 / \sqrt{n}$ ou $1 / n$;

- Sem ou Com Decomposição de Cholesky.

2. Preditor-Corretor:

- Foram usados os mesmos parâmetros do Primal-Dual, com a única diferença que apenas a segunda fórmula de $\mu$ foi usada. 
Isto totaliza 72 versões para o MPI primal-dual e 36 para o MPI preditorcorretor, somando 108 versões de métodos de pontos interiores. Como testamos cada uma das 108 versões para os 10 exemplos do PIPEFA, teríamos 108 tabelas com informações sobre tempo, número de iterações e número de operações de ponto flutuante.

Para maior clareza na apresentação dos resultados, calculamos para cada versão o percentual de problemas testes que convergiram. Agrupamos as médias de acordo com o método utilizado, o uso de decomposição de Cholesky e estrutura esparsa considerada, obtendo a Tabela 2.

\begin{tabular}{|c|c|c|c|}
\hline Matriz & Método & Cholesky & Convergência(\%) \\
\hline \multirow[t]{4}{*}{$A$} & \multirow[t]{2}{*}{ Primal-dual } & Não & 96,66 \\
\hline & & Sim & 88,33 \\
\hline & \multirow[t]{2}{*}{ Preditor-corretor } & Não & 95,00 \\
\hline & & Sim & 100,00 \\
\hline \multirow[t]{4}{*}{$A$ e $S$} & \multirow[t]{2}{*}{ Primal-dual } & Não & 73,33 \\
\hline & & Sim & 72,50 \\
\hline & \multirow[t]{2}{*}{ Preditor-corretor } & Não & 90,00 \\
\hline & & Sim & 90,00 \\
\hline \multirow{4}{*}{$A_{i}$ e $S_{i}$} & \multirow[t]{2}{*}{ Primal-dual } & Não & 93,33 \\
\hline & & Sim & 93,33 \\
\hline & \multirow[t]{2}{*}{ Preditor-corretor } & Não & 100,00 \\
\hline & & Sim & 100,00 \\
\hline
\end{tabular}

Tabela 2: Resultados resumidos de convergência.

Pela Tabela 2, observamos que o método preditor-corretor com decomposição de Cholesky apresentou o melhor desempenho. Portanto, para comparar o desempenho do MPI de acordo com o uso de estrutura esparsa, utilizamos os resultados para o preditor-corretor com decomposição de Cholesky para as 3 estruturas de $A$ e com $\tau=0.995, \sigma=1 / n$ e a segunda fórmula de $\mu$. Os resultados estão na Tabela 3.

A Tabela 3 fornece os valores médios do desempenho dos 3 métodos com diferentes estruturas esparsas aplicados aos 10 problemas testes do PIPEFA. Os testes foram realizados em MATLAB 5.2 em uma estação SUN ULTRA 1.

Vale destacar que, o comando linprog do MATLAB, que utiliza o método preditorcorretor, não convergiu para o arquivo Exemp13. Esse resultado ocorreu tanto na versão 5.2 como na versão 6.0 .

\begin{tabular}{|c|c|c|c|}
\hline Método & Tempo Médio & Média Iterações & Média Flops \\
\hline Matriz A & 0,7394 & 12,1818 & 9365907 \\
Matrizes A e S & 0,9108 & 11,2727 & 6946843 \\
Matrizes Ai Si & 1,4121 & 11,3636 & 3629597 \\
\hline
\end{tabular}

Tabela 3: Desempenhos médios de convergência para o preditor-corretor. 


\section{Conclusões}

Em relação a velocidade, como podemos verificar pela Tabela 3, quanto mais o método aproveita a estrutura esparsa do problema, menor será o número médio de operações de ponto flutuante(flops). A justificativa para o aumento do tempo médio é devido a utilização de laços (for, while, etc) nestes métodos, o que em MATLAB os torna mais lentos. Porém, pretendemos, em trabalhos futuros utilizar uma linguagem de programação como $\mathrm{C}++$, Java, FORTRAN, e acreditamos que teremos um tempo médio que será de acordo com número médio de flops. Ou seja, o método que explora mais a esparsidade será muito mais rápido. Quanto a robustez, observamos que o método preditor-corretor com maior aproveitamento da estrutura esparsa funcionou para todos arquivos do PIPEFA, quaisquer fossem os parâmetros considerados, inclusive para $\sigma=0$, sendo mais robusto que os demais métodos e que o comando linprog do MATLAB.

\section{Agradecimentos}

Este trabalho contou com o suporte financeiro da Fundação de Amparo à Pesquisa do Estado de São Paulo (FAPESP) e do Conselho Nacional de Desenvolvimento Científico e Tecnológico (CNPq).

\section{Referências}

[1] J. Castro, A Specialized Interior-Point Algorithm for Multicommodity Network Flows, SIAM J. Optimization, 10 (2000), 852-877.

[2] M.F. Carvalho, C.A.O. Fernandes e P.A.V. Ferreira, Multiproduct multistage production scheduling (MMPS) for manufacturing systems, Production Planning Control, 10 (1999), 7, 671-681.

[3] M. F. Carvalho and F. E. Pereira, Cooperative Enterprise Platform In, IFAC Workshop on Manufactoring Systems: Moddeling Management and Control Viena, I (1997), 313-318.

[4] J.L. Kennington, A Survey of Linear Cost Multicommodity Network Flow, Operations Research, 26 (1978), 2, 209-236.

[5] A. Yamakami, M.T. Takahashi e M.F.Carvalho, Comparison of Some Algorithms for Manufacturing Production Planning, in "IFAC-MIM 2000 Symposium on Manufacturing, Modeling, Management and Control", 2000, 280-284.

[6] S.J. Wright, "Primal-Dual Interior-Point Methods", SIAM Publications, SIAM Philadelphia, PA, USA, 1996. 